\title{
AR-RBFS: Aware-Routing Protocol Based on Recursive Best-First Search Algorithm for Wireless Sensor Networks
}

\author{
Farzad Kiani \\ Computer Engineering Department, Engineering and Natural Sciences Faculty, Istanbul Sabahattin Zaim University, \\ 34303 Istanbul, Turkey
}

Correspondence should be addressed to Farzad Kiani; farzad.kiani@izu.edu.tr

Received 25 May 2016; Accepted 14 July 2016

Academic Editor: Fei Yu

Copyright (C) 2016 Farzad Kiani. This is an open access article distributed under the Creative Commons Attribution License, which permits unrestricted use, distribution, and reproduction in any medium, provided the original work is properly cited.

Energy issue is one of the most important problems in wireless sensor networks. They consist of low-power sensor nodes and a few base station nodes. They must be adaptive and efficient in data transmission to sink in various areas. This paper proposes an aware-routing protocol based on clustering and recursive search approaches. The paper focuses on the energy efficiency issue with various measures such as prolonging network lifetime along with reducing energy consumption in the sensor nodes and increasing the system reliability. Our proposed protocol consists of two phases. In the first phase (network development phase), the sensors are placed into virtual layers. The second phase (data transmission) is related to routes discovery and data transferring so it is based on virtual-based Classic-RBFS algorithm in the lake of energy problem environments but, in the nonchargeable environments, all nodes in each layer can be modeled as a random graph and then begin to be managed by the duty cycle method. Additionally, the protocol uses new topology control, data aggregation, and sleep/wake-up schemas for energy saving in the network. The simulation results show that the proposed protocol is optimal in the network lifetime and packet delivery parameters according to the present protocols.

\section{Introduction}

Wireless ad hoc networks are considered a great development in the autonomous systems technology. They are divided into two big general categories so one of them is wireless sensor networks (WSNs). These networks have played an important role in the development of intelligent systems because they are cheap, scalable, and flexible. These networks include large numbers of inexpensive, small-size sensor nodes and a few base stations (BS) or sinks so the sensor nodes have limited battery, throughput, and memory [1] and sensitive channel controls [2]. These nodes with low-powered sensing devices equipped for mobilization are deployed on animals, birds, plants, or humans to achieve better targeting and data fidelity. They form a wireless network by creating a communication system amongst themselves. Then, the sensor nodes sense and process a phenomenon and send their results to the BS or sink. WSNs can be used in different applications such as military applications, forests, monitoring systems, agriculture, and medicine. Despite the various applications of these networks, they suffer from resource constraints as energy and memory. Therefore, researchers focus more on the constraints and balancing between resources and goals of system issues when they want to design any model for WSNs applications. One of the most important constraints is energy matter [3]. There are two types of energy consumption between sensor nodes. The first is energy consumption in communications and the second is consumption in computations. Obviously, communications consume more energy according to consumption computations. Therefore, minimizing communication costs is an important issue. Researchers propose different approaches to this goal such as energy efficiency by routing techniques, data aggregation, and duty cycle techniques. Sometimes, there are conflicts between some system targets such as reliability and energy saving. If the system designer wants high reliability performance, the sensor nodes will waste more energy. Therefore, designers must be careful about the balancing issue in the network [3].

The communication architecture and stack protocol of WSN have five layers and three planes $[1,4]$. These layers are 


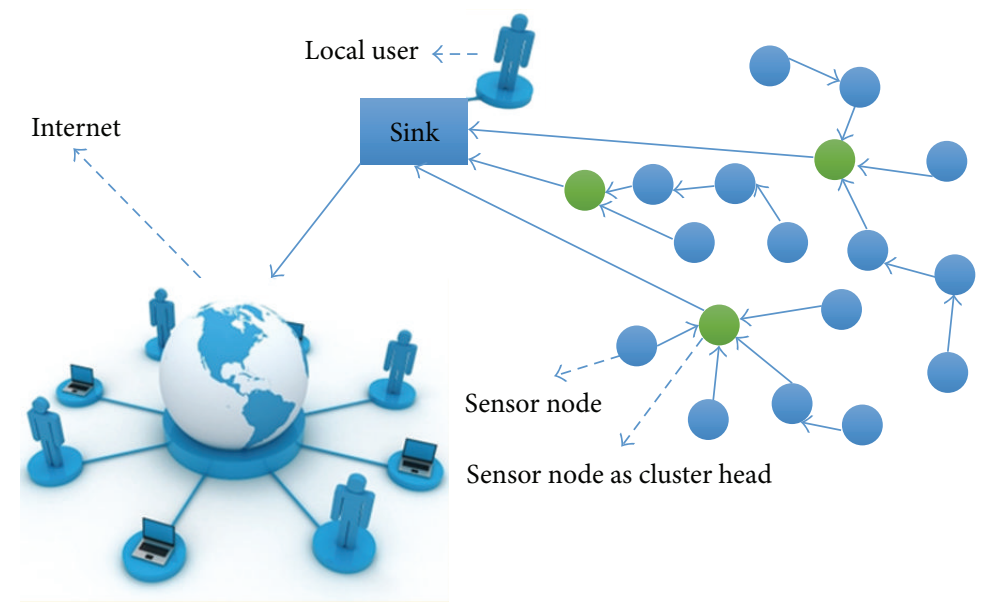

FIgURE 1: Data transmission view in WSN [4].

physical layer, data link layer, network layer, transport layer, and application layer. The planes are power management plane, mobility management plane, and task management plane. One important layer is the network layer. The network layer handles collected data from source node to destination node. Routing protocols in WSNs are different than traditional routing protocols in several respects. For example, they do not use ID-addresses or fix routes schemas in the routing processes. Therefore, the IP-based routing protocols cannot be used in the WSNs. A routing protocol should be attending to the limitations of network resources as energy, communication bandwidth, memory, and computational capabilities. Proper management of these limitations can increase the network lifetime. Additionally, a routing protocol can consider faulting tolerance, latency, data aggregation, energy efficiency, and providing internetworking with external networks-gateway principles $[3,6]$. It should be noted that the data link layer handles how two nodes talk to each other and the network layer is responsible for deciding which node talks [4].

Many tasks of the above layers can be achieved by designing an aware-routing protocol and considering the importance of the balance issue between system goals and resources. In this paper, we proposed a new aware-routing protocol based on recursive searching method and implemented it for monitoring agriculture and university environments. One of the important goals in the routing algorithms is energy efficiency where the selective paths can increase network lifetime. In most applications, sensor nodes can aggregate data and send reached packets to the BS/sink. In the multihop networks, some of the sensor nodes are intermediate nodes for data transmission between two nodes. Figure 1 shows a sample of data forwarding in the cluster-based network. These collaborator nodes are called intermediate nodes. The method of finding optimized paths and selection of the intermediate nodes are the major tasks of routing protocols.

The rest of the paper is organized as follows. Section 2 describes related works in routing protocols on wireless sensor networks. Section 3 details the structure of the proposed protocol. In Section 4, we listed the simulation parameters

\section{Routing protocols classification on wireless sensor networks}

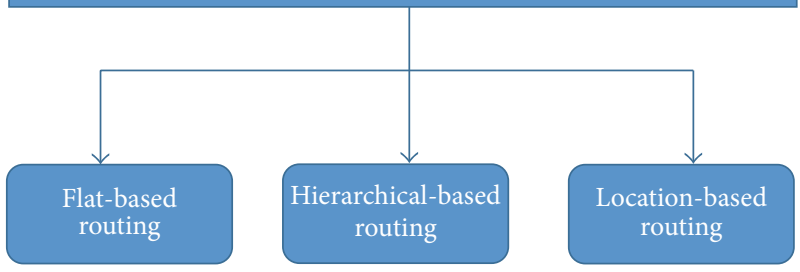

FIGURE 2: Routing protocols classification for WSNs [4].

and analysis of the results. Finally, conclusion is given in Section 5.

\section{Relative Works}

Many current algorithms and routing protocols can be classified into three groups as shown in Figure 2.

In the flat-based routing, the nodes forward self-data to the other nodes or BS/sink by single or multihop methods. Each sensor node has the same role in the network. As mentioned, ID is not used in the WSN structures because they consist of large numbers of nodes. Therefore, BS/sink will wait for receiving an answer from the sensor nodes when it broadcasts any query to all or selected regions of the network [8]. Accordingly, redundancy in the whole network is high and it causes more consumption of energy. This problem can be solved by data aggregation technique [1]. The flat-based routing study case is proposed in many protocols such as SPIN [9], Directed Diffusion (DD) [5], EAD [10], and GBR [11].

The flat-based routing protocols are most suitable for specific applications such as event detection. If this type of routing protocol is applied in the monitoring-based applications, then accuracy of the data is somewhat doubtful and it does not represent a real condition of the environment in transmission data packets. This problem or transmission delays in the large-scale networks are not acceptable. Hence, the flat-based routing protocols consume significant energy because a large number of nodes participate in the data transmission phase 


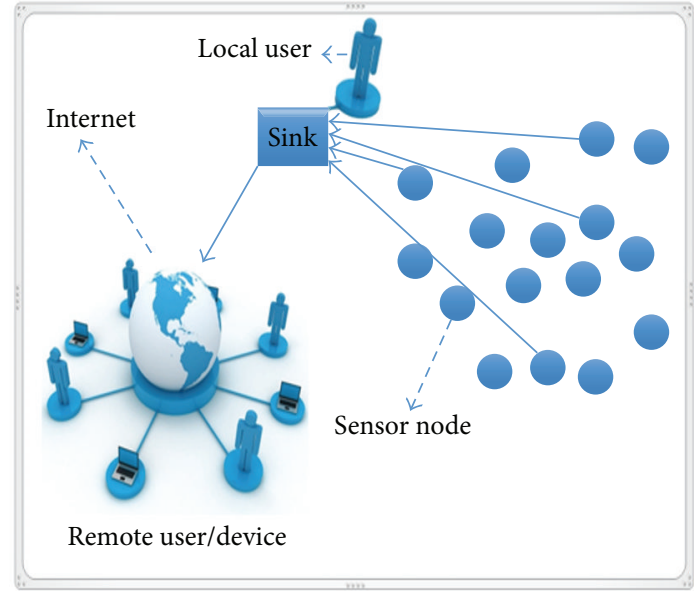

(a) Single hopping

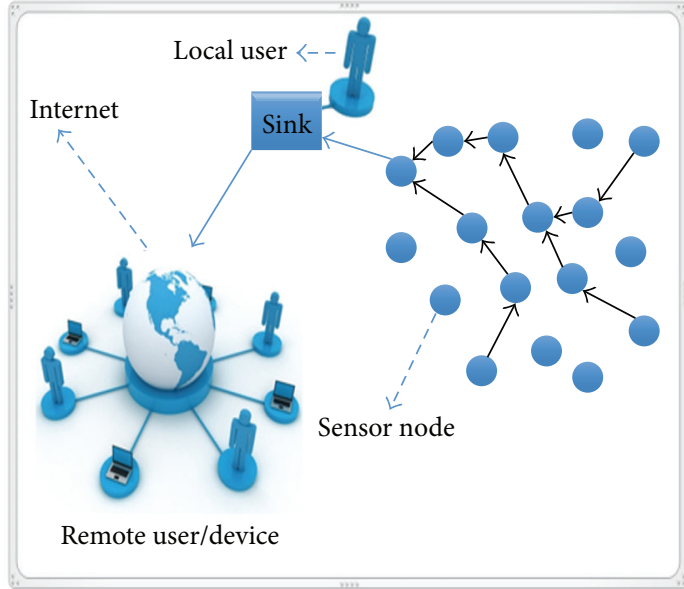

(b) Multihopping

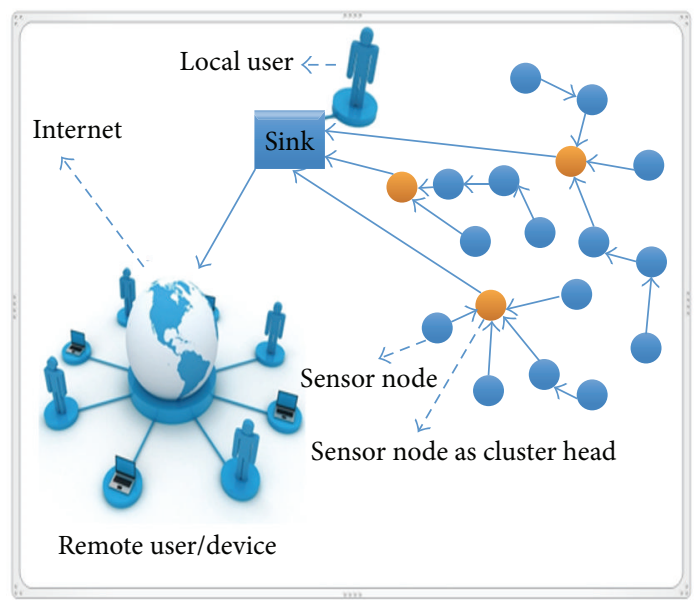

(c) Hierarchical-based routing

FIGURE 3: Models of routing and data transmission protocols [5].

and any node has the same role in the network. In Figure 3, we presented the three models of routing techniques. In model A, each node sends its sensed data to the sink directly without intermediate nodes. This position causes wasting energy in the whole network. Model B is the improvement of flat-based routing techniques that uses multihop method for sending data or responding to requested queries. This model reduces energy consumption significantly but, in the general case, it cannot reach energy efficiency yet. Researchers work on the energy efficiency factor in the hierarchically based routing protocols more than other protocols. They offered a new model for network configuration and named it hierarchicalbased routing algorithms. This paper proposes an awarerouting protocol based on the hierarchical method. The basis of our approach is to cluster and use $\mathrm{CH}$ nodes as an interface between the clusters. $\mathrm{CH}$ nodes can communicate with other $\mathrm{CHs}$ or sink except for the nodes that are in the same cluster. Hence, sensor nodes are not involved in retention of routes between source nodes and sink. Generally, CHs use a CSMA MAC method and each node chooses a cluster to which it will belong and it is calculated based on the received signal strength or its distance to the $\mathrm{CH}$ node. Under the same conditions, a node randomly selects a $\mathrm{CH}$ node and cluster. $\mathrm{CH}$ nodes compute a TDMA schedule for data transmission in clusters. After that, each node will know how and when it can send its data to other members. The schedule is broadcast to all nodes in the cluster except the $\mathrm{CH}$ node. The sensor nodes will send their data packets to $\mathrm{CH}$ nodes after formation clusters and allocation schedule mechanisms [6]. The network lifetime is increased and the routing protocols are converted to energy efficiency methods in the WSNs. Many protocols are proposed in the category such as HEEDNPF [12], Improved-LEACH [13], FTIEE [3], New-LEACH [14], EE-LEACH [15], and SALMA [16].

In the WSNs, almost every routing protocol needs the location information of sensor nodes. It is calculable from the distance between two special nodes. Coordinates of neighboring nodes were obtained using exchanges of some information between neighbors. If addressing scheme for a sensor network is not known, an energy efficient routing protocol can be accessible. Almost, the location-based routing protocols are proposed in the MANET [17]. However, 


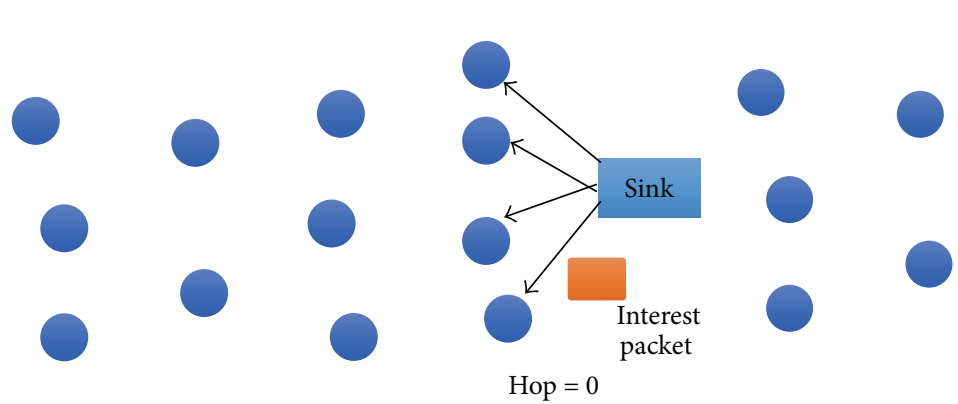

(a)
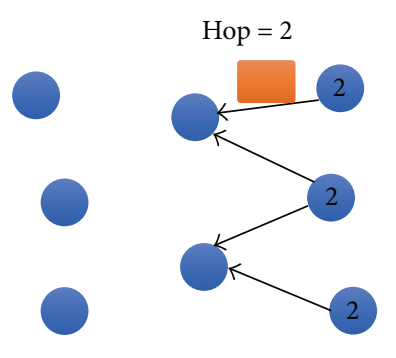

(c)

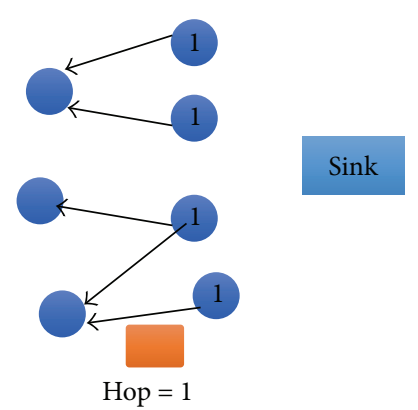

(b)

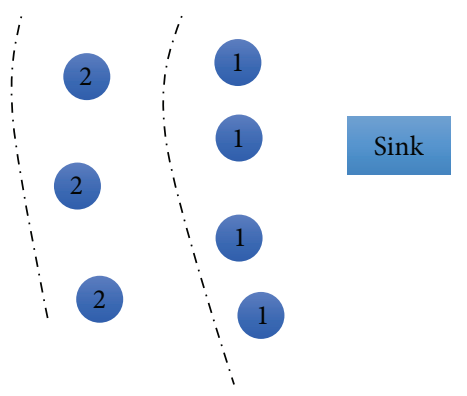

(d)

Figure 4: The network deployment.

they are also well applicable to WSNs where there is less or no mobility. The location of nodes can be reachable by communicating with a GPS or satellite directly but they are not inexpensive. More energy saving in the location-based protocols can be obtained by maintaining more nodes in sleep mode. The problem of scheduling of sleep period designing for each node in a localized manner was addressed in [6]. Many protocols are proposed in this category such as GAF [18], GEAR [19], and EEAR [20].

\section{Designing Aware-Routing Protocol Based on Recursive Best-First Search Algorithm (AR-RBFS)}

In the proposed protocol, data transmission is based on hybrid model and routes discovery is based on query model [6]. The paths which have been found by the protocol are related to destination nodes that are selected via the sink. Then, data can be collected by time-driven, query-based, or event-based methods. Additionally, a hybrid model is possible. For example, the time-driven methods in surveillance application, the event-based methods in target tracking in army applications, and the query-driven methods in habitat monitoring applications can be used. In the time-based approach, every node sends data packets periodically. In the event- and query-based approaches, if an event happens or a query is sent via the BS/sink to all sensor nodes, data transmission will be realized in the network. Data delivery is one of the important parameters in routing algorithms. The proposed protocol is implemented in two phases and discussed below.
3.1. Network Deployment Phase. Communications between sensor nodes must be clear in the network after completing the distribution of sensors in an environment. In the first phase, the sensor nodes will be deployed in the environment and they will be run in the whole network. In the second phase, condition-based environments may require the redeployment of nodes due to a high likelihood of continuous changes of network structures, topologies, and redeployment issue. The network structure is cluster-based and it will be shown as grid. Deployment of the network is done according to the following method. This method is valid and it is the same for both case studies of environments. Sink broadcasts interest message packet to the sensor nodes after deployment of the sensor nodes in the environment. This packet includes the interest hop field that has a default value of zero. The sensor nodes receive the packet and then each node adds one unit to interest hop value and saves this value as selfinterest hop. Nodes that have the same interest hop value will be placed in the same layer. When the sink broadcasts the packet, it is only received by neighbors of the sink and then these neighbors broadcast it to their neighbors. This process is to be continued until all sensor nodes receive the packet. If the sensor nodes with interest hop value get any interest packet again, they remove the packet. Figure 4 shows the first phase of the proposed protocol. The nodes placement in the virtual layers is based on the closeness to the sink and number of hops. The first phase is finished in step $d$ so all layers were created in the network. The pseudocode of network deployment is shown in Algorithm 1.

3.2. Data Transmission Phase. Our proposed protocol (ARRBFS) uses data-centric routing schema to flow data packets 
(1) Sink broadcast interest-message to all nodes

(2) The nodes receive the message

(3) For all nodes

(4) If message $=$ not repeat

(5) Interest hop $=$ interest hop +1

(6) Else go to (7).

(7) For all nodes

(8) If interest hop's nodes are same

(9) They are in a same layer

(10) For all layers

(11) While (duty-task-message $\leq$ our limited number)

(12) Send duty-task-message from any node to self-neighbors

(13) If receive-answer-count $=$ our limited number

(14) While (Time is true)

(15) Node is sleep mode

(16) Go to (12).

Algorithm 1: Pseudocode of network deployment.

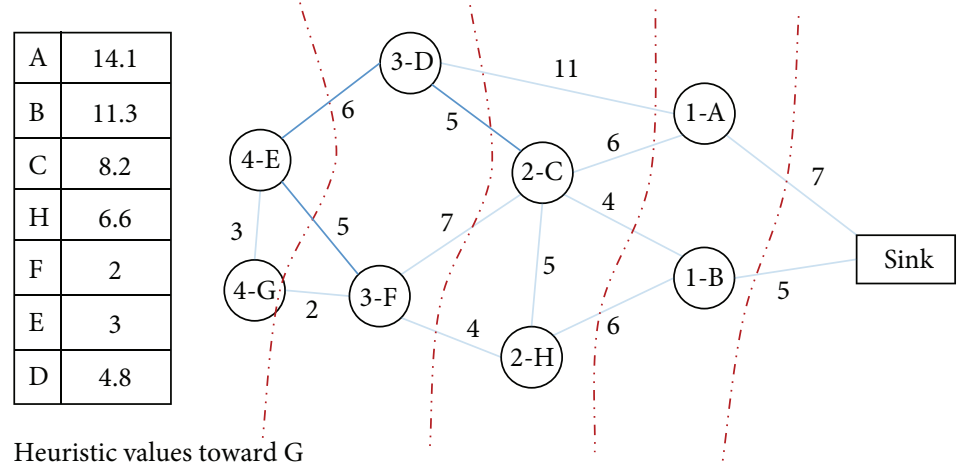

FIgURE 5: Routing and data transmission.

through multiple layers to the sink with route discovery. It is based on aware searching algorithm. Suppose we deployed our network and destination node is G as shown in Figure 5. This node is chosen via sink and is informed to network nodes based on a query. In this case, heuristic value $\left(h_{n}\right)$ is calculated to all nodes and is saved for each node routing table as temporary. Each query message packet has certain lifetime that is assigned by the sink. This technique can avoid loops and energy and bandwidth wasting. Heuristic values are calculated based on direct distance costs per node to destination nodes.

This phase of the AR-RBFS has two different versions. One of them is applicable in chargeable environments. It is shown in (1). It creates a tree based on recursive search method for finding the best route between sink and destination node. It has similarity to deep search algorithms. The aim of the phase is to find the best neighbors and routes to destination nodes. If the application environment has electrical installations, we do not have an energy problem. In this case, our algorithm is run on first scenario as described in (1). In this scenario, the recursive algorithm is used in the protocol that allows the sensors to keep optimizing by using self-memory. Our algorithm selects a better route in each step and also maintains the second best route (not all routes). A sample of all steps of the route discovery algorithm are shown in Figure 6. The steps are the same in the two versions and only the heuristic values of the calculations are different from each other. Calculation of the heuristic value for the second scenario is based on (2). Consider the following:

$$
f\left(n_{i}\right)=h\left(n_{i}\right)+g\left(n_{i}\right),
$$

where $h(n)$ is for heuristic costs and $g(n)$ is for path costs used. For example, in Figure 5, $g(n)$ is 6 between $1-\mathrm{B}$ and $2-\mathrm{H}$ and heuristic cost from B to destination node $(G)$ is 11.3 .

If the heuristic value is admissible, this proposed algorithm will be robust. However, it still has a problem in the over node regeneration because it has limited memory. ClassicRBFS [7] algorithm expands nodes in the best-first order even with a nonmonotonic cost function. The Classic-RBFS is a recursive algorithm that uses the heuristic and $g(n)$ values. $g(n)$ is the cost of path from root to " $n$." The ClassicRBFS is better than similar methods. Pseudocode is shown in Algorithm 2.

When the application environment does not have an energy problem, we can use the Classic-RBFS algorithm in 


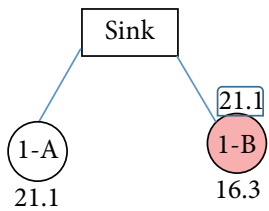

Fringe list: $\{1-\mathrm{B}(16.3), 1-\mathrm{A}(21.1)\}$

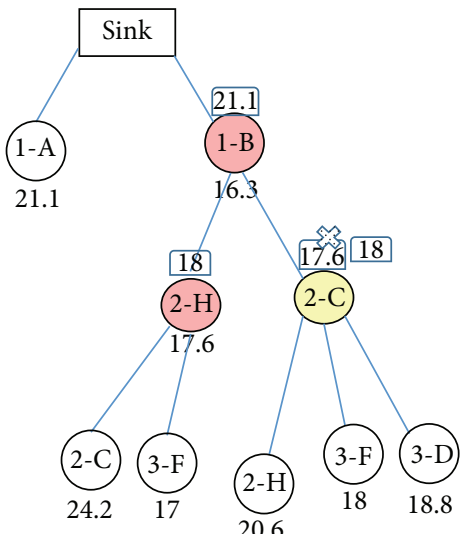

Fringe list: $\{3-F(17), 3-F(18)\}$

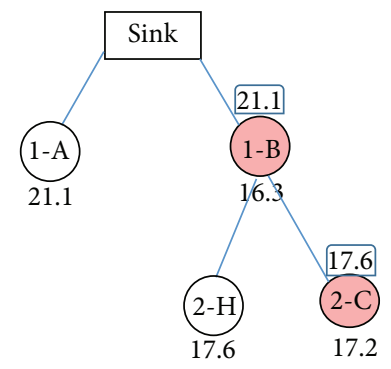

Fringe list: $\{2-\mathrm{C}(17.2), 2-\mathrm{H}(17.6)\}$

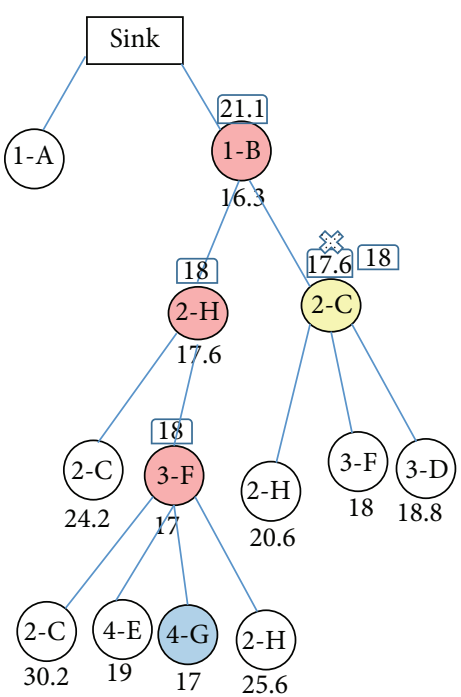

Fringe list: $\{4-\mathrm{G}(17), 3-\mathrm{F}(18)\}$

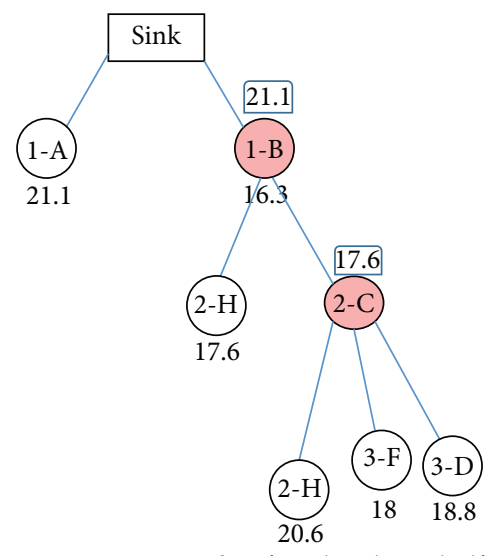

Fringe list: $\{2-\mathrm{H}(17.6), 3-\mathrm{F}(18)\}$

\footnotetext{
Fringe list is a sorted array

Length of the list always is two $\rightarrow$ energy and memory saving

(1) Best $\rightarrow \rightarrow$ select and (2) best $\rightarrow-\rightarrow$ maintain in selected node

Selected node

Cancelling the selected node

Goal node (destination node)
}

FIGURE 6: AR-RBFS algorithm steps.

routes discovery with some modification as described [7]. But it cannot be used in the environments that have energy problems. Therefore, we need another approach for routes discovery. In this case, our algorithm uses intermediate nodes by new $f$ function given in (2). In this equation, we must consider the energy level of each sensor node $\left(E\left(n_{i}\right)\right)$. Consider the following:

$$
f\left(n_{i}\right)=\frac{\left[h\left(n_{i}\right)+g\left(n_{i}\right)\right]}{E\left(n_{i}\right)} .
$$

Moreover, nodes have to be of a certain amount of energy to communicate. For example, if energy of any node is more than $1 \mathrm{nW}$, then the node can communicate with its neighbors based on (2). Otherwise, the nodes cannot collaborate in the routes searching process. Therefore, the energy issue is important in the environments that are difficult to charge. Energy saving of the AR-RBFS protocol is based on duty cycle approaches. In this state, all nodes of each virtual layer can be modeled as a random graph [21]. This approach is based on duty cycle methods. The concept of the duty cycle methods is the maintenance of minimum active nodes for energy efficiency [22]. In fact, duty cycling methods focus on the networking subsystem. A good way for energy saving is to use the sleep (low-power) mode for idle sensor nodes. The proposed protocol creates a full connection topology for all active nodes in every layer. This protocol uses a virtual layers structure and it attempts to keep the network connections by maintaining enough number of sensor nodes in active mode in each layer as delegate nodes. These active nodes will have 

$\operatorname{RBFS}(n, B)$
(1) if $n$ is a goal
(2) solution $\leftarrow n$; exit ()
(3) $C \leftarrow \operatorname{expand}(n)$
(4) if $C$ is empty, return $\infty$
(5) for each child $n_{i}$ in $C$
(6) if $f(n)<F(n)$ then $F\left(n_{i}\right) \leftarrow \max \left(F(n), f\left(n_{i}\right)\right)$
(7) else $F\left(n_{i}\right) \leftarrow f\left(n_{i}\right)$
(8) $\left(n_{1}, n_{2}\right) \leftarrow$ best $_{F}(C)$
(9) while $\left(F\left(n_{1}\right) \leq B\right.$ and $\left.F\left(n_{1}\right)<\infty\right)$
(10) $\quad F\left(n_{1}\right) \leftarrow \operatorname{RBFS}\left(n_{1}, \min \left(B, F\left(n_{2}\right)\right)\right)$
(11) $\quad\left(n_{1}, n_{2}\right) \leftarrow$ best $_{F}(C)$
(12) return $F\left(n_{1}\right)$

Algorithm 2: Pseudocode of general RBFS algorithm [7].

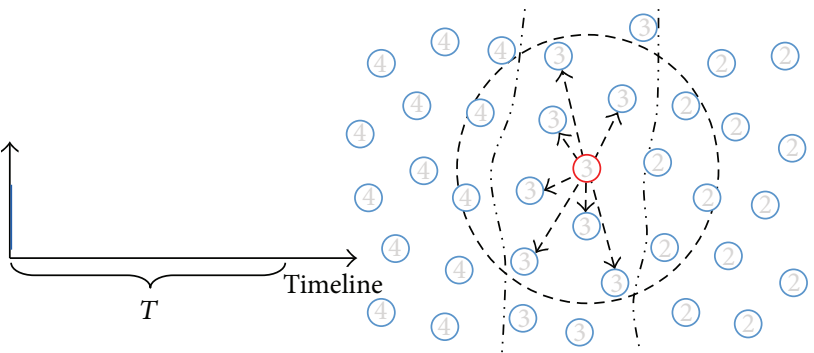

FIgURE 7: The selected node sends HELLO message to self-layer members.

communication with their sleep neighbor nodes in the same layer. In accordance with this purpose, the protocol uses two parameters. One of them is $T$ that shows period times of duty cycling. Another variable is $C$ and it defines the internal communications degree. Each node can go to sleep mode when it receives $C$ numbers of task change messages from its active neighbors. The proposed approach in the paper is based on topology control technique and it uses some parameters for efficient data transmission.

Numbers of active nodes and communications in the network are increased with increasing the value of $C$ parameter. In order to do so, each node spot considers time periods with duration $T$. Any node is waiting during $t_{v}$ times and it is in sleep mode in this time. $t_{v}$ is distributed uniformly and its value is in the range $[0, T)$. After this time, the node converts to active mode and sends HELLO message to selfneighbor. It goes into sleep mode again upon receiving the responses from the neighbors. The responses are based on $C$ value and time is different to nodes. The $C$ and $T$ parameters guarantee connectivity of the network. Each active sensor node sends HELLO message packets to its sleep neighbors (Figure 7). Then, it listens to HELLO messages sent by other nodes. Assuming that the value of $C$ parameter is three, we illustrate the operating style of the algorithm in Figure 8. The values of $C$ and $T$ are related to our environment conditions and parameters and also our system goals. In fact, a node will

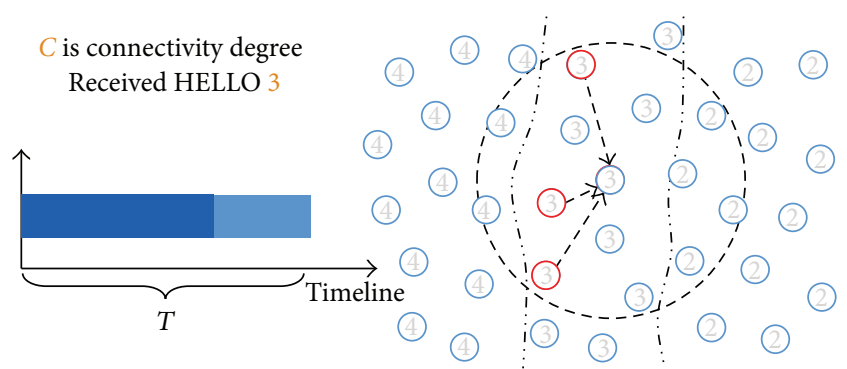

FIGURE 8: The received HELLO packets illustrate activation of other nodes (in this example, $C$ is given the value 3 ).

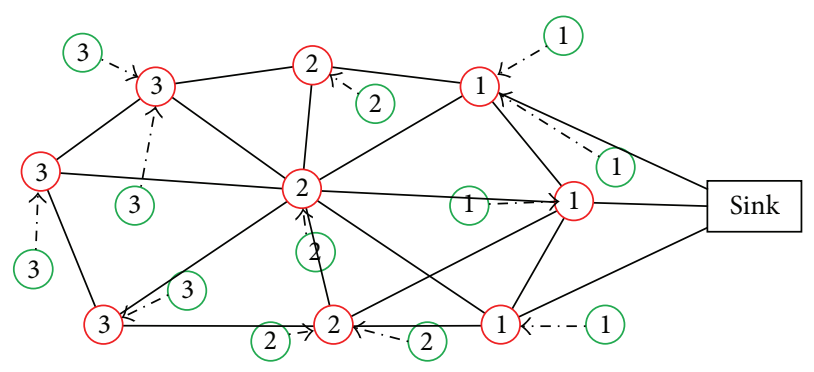

FIGURE 9: Packet routing in sleeping nodes.

be in the sleep mode after receiving an answer from the three neighboring nodes in the same layer.

Each node has waiting time $\left(t_{v}\right)$ and period time $(T)$. When it receives the activation HELLO message from its neighbors, it converts its mode to active. The active nodes will be in the activation mode for $T$ time. The activation message numbers are reached from $C$ parameter. For example, in Figure 8, value of $C$ is " 3 ." Indeed, each node after publishing its activity sends its own HELLO packet and then goes to sleep mode after finishing its periods.

In this scenario, sleep nodes cannot be in the route discovery process by AR-RBFS algorithm but these nodes can send received packets to their neighbors active nodes when they become active. The received mode of sensor nodes is active any time even when the node is in the sleep mode. The scenario is shown in Figure 9.

\section{Simulation}

In the simulation of AR-RBFS protocol, AR-RBFS protocol results are compared with HEED-NF, EE-LEACH, and SALMA protocols. The output parameters are network lifetime and average packet delivery. Also, the input parameters are initial energy of each sensor node, radio and sensor energy consumption, transmit and receive/sense process costs, and buffer size (Table 1). In the simulation scenario, the sensor nodes are not mobile and they have similar architecture and design fundamental. The sensor nodes collect sensing phenomena and forward them toward BS/sink via intermediate nodes as multihopping method. A snapshot of AR-RFBS is shown in Figure 10. In this figure, the virtual layer 
TABLE 1: Values of input parameters.

\begin{tabular}{lccc}
\hline Initial (max) energy & $0.7 \mathrm{~J} / \mathrm{bit}$ & Receive buffer size & 10000 bytes \\
Radio/sensor energy consumption & $40 \mathrm{~nJ} / \mathrm{bit}$ & Send buffer size & 10000 bytes \\
Transmit process cost & $40 \mathrm{~nJ} / \mathrm{bit}$ & Deployment area size & $(600 \times 600) \mathrm{m}$ \\
Receive/sense process cost & $10 \mathrm{~nJ} / \mathrm{bit}$ & Send/receive buffer counts & 20 \\
Data packet size & $500 \mathrm{bytes}$ & Sink position & $(600 \times 300) \mathrm{m}$ \\
Sensing radius & $7.5 \mathrm{~m}$ & Transmission radius & $15 \mathrm{~m}$ \\
\hline
\end{tabular}

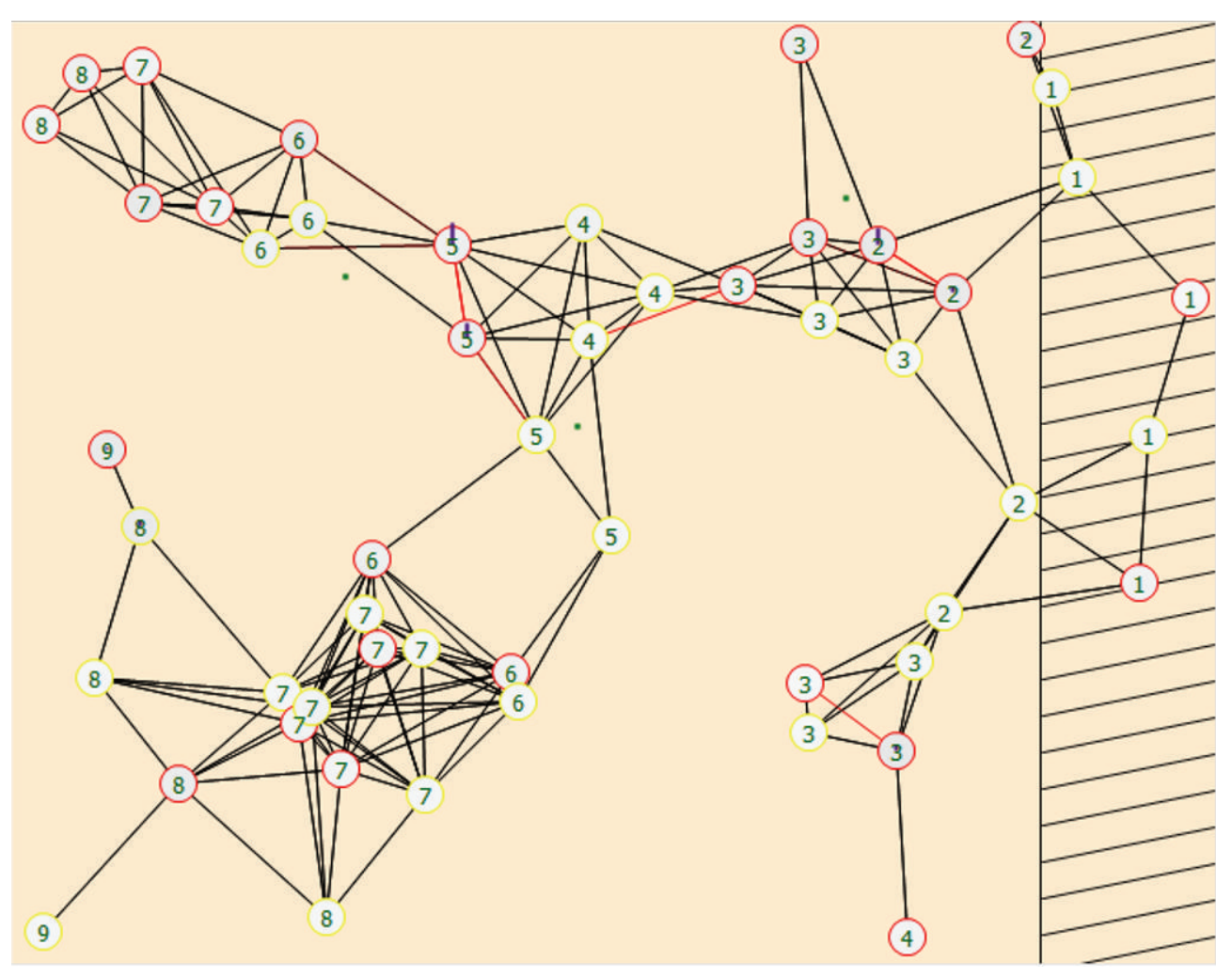

FIGURE 10: A snapshot of basic network topology in AR-RBFS protocol.

approach can appear. The proposed protocol can be available in areas such as border security.

The simulations are based on two different scenarios. The first scenario is developed for the nodes that have an unlimited energy source or easy-to-recharge batteries. They are charged by the environment energy source such as electrical installations. Another scenario is for low-power energy source environments.

The results show that AR-RBFS has a good performance in the network lifetime and average packet delivery rate. It can increase the energy saving factor using data aggregation, topology control, and sleep/wake-up methods. Network lifetime is directly related to the number of nodes. The network lifetime will be better when we use more sensor nodes. We can say that, with the increasing number of nodes, the number of received packets is increased but packet delivery rate is almost reduced because some of the factors such as increasing density of nodes, hop counts, and node failure probability are effective. Figures 11 and 12 show simulation results on three protocols and proposed two scenario-based protocols.

\section{Conclusion}

This paper proposed the design of an AR-RBFS based routing protocol in two different scenarios on WSN. It is used to evaluate the power consumption and packet delivery rate of wireless sensor nodes. The algorithm computes an optimized path to route the packets from the sink to the destination node. The cluster-based structure and using the new duty cycle based energy saving technique help the network have a good performance in the output parameters (network lifetime and average packet delivery rate). Since the packets are routed through an optimized path, the energy required for transmission will be decreased; thus, the lifetime of the system is increased. Simulation results show that the proposed 


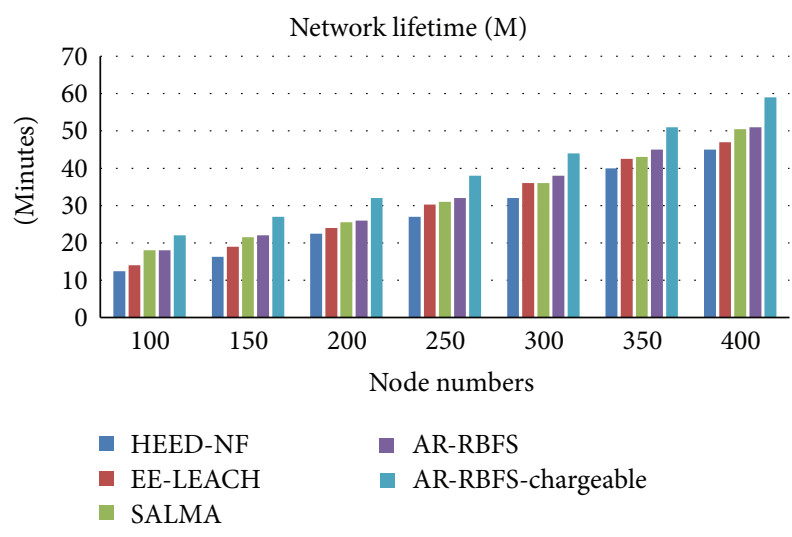

FIGURE 11: Network lifetimes in AR-RBFS (2 scenarios), HEED-NF, EE-LEACH, and SALMA.

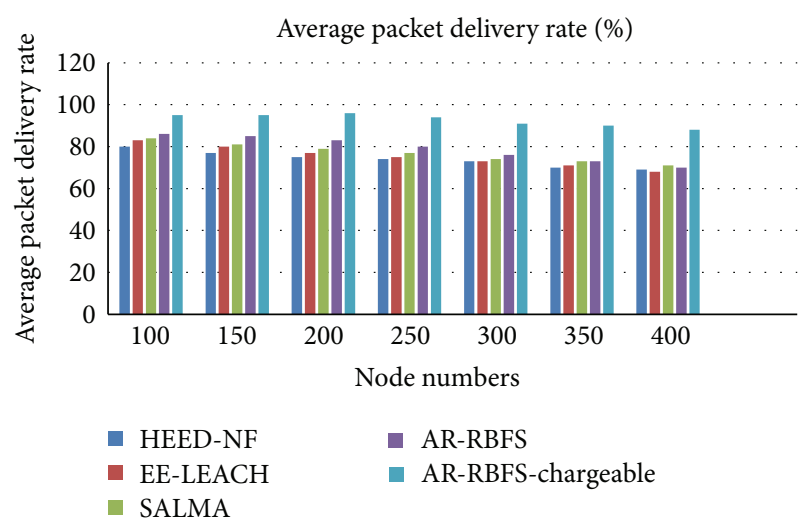

FIGURE 12: Average packet delivery rates in AR-RBFS (2 scenarios), HEED-NF, EE-LEACH, and SALMA.

AR-RBFS based approach for improving the energy saving in the WSN is better than the SALMA, EE-LEACH, and HEEP$\mathrm{NF}$ routing algorithms. Hence, the proposed algorithm is better than the existing algorithms. The proposed protocol for both environments can be implementable on various applications such as agriculture.

\section{Competing Interests}

The author declares that there are no competing interests.

\section{References}

[1] I. F. Akyildiz, W. Su, Y. Sankarasubramaniam, and E. Cayirci, "Wireless sensor networks: a survey," Computer Networks, vol. 38, no. 4, pp. 393-422, 2002.

[2] F. Kiani, "A novel channel allocation method for time synchronization in wireless sensor networks," International Journal of Numerical Modelling: Electronic Networks, Devices and Fields, vol. 29, no. 5, pp. 805-816, 2016.

[3] F. Kiani, E. Amiri, M. Zamani, T. Khodadadi, and A. Abdul Manaf, "Efficient intelligent energy routing protocol in wireless sensor networks," International Journal of Distributed Sensor Networks, vol. 2015, Article ID 618072, 13 pages, 2015.
[4] I. F. Akyildiz, W. Su, Y. Sankarasubramaniam, and E. Cayirci, "A survey on sensor networks," IEEE Communications Magazine, vol. 40, no. 8, pp. 102-105, 2002.

[5] C. Intanagonwiwat, R. Govindan, and D. Estrin, "Directeddiffusion: a scalable and robust communication paradigm for sensor networks," in Proceedings of the 6th Annual International Conference on Mobile Computing and Networking (MobiCom '00), pp. 56-67, ACM, 2000.

[6] F. Kiani, Designing New Routing Algorithms Optimized for Wireless Sensor Network, LAP LAMBERT Academic Publishing, Dusseldorf, Germany, 2014.

[7] M. Hatem, S. Kiesel, and W. Rosel, "Recursive best-first search with bounded overhead," in Proceedings of the 29th AAAI Conference on Artificial Intelligence, vol. 29, pp. 1152-1157, 2015.

[8] M. Maroti, "Directed flooding-routing for wireless sensor network," in Proceedings of the ACM/IFIP/USENIX International Conference on Middleware, vol. 2, pp. 99-114, 2004.

[9] A. Martorosyan, A. Boukerche, and R. W. Pazzi, "A taxonomy of cluster-based routing protocols for wireless sensor networks," in Proceedings of the International Symposium on Parallel Architectures, Algorithms, and Networks (i-span '08), pp. 247253, Sydney, Australia, May 2008.

[10] R. C. Shah and J. M. Rabaey, "Energy aware routing for low energy ad hoc sensor networks," in Proceedings of the IEEE Wireless Communications and Networking Conference (WCNC '02), pp. 350-355, Orlando, Fla, USA, March 2002.

[11] L. Miao, K. Djouani, A. Kurien, and G. Noel, "A competing algorithm for gradient based routing protocol in wireless sensor networks," in Proceedings of the International Conference on Wireless Information Networks and Systems (WINSYS '10), pp. 82-89, Athens, Greece, July 2010.

[12] H. Taheri, P. Neamatollahi, M. Naghibzadeh, and M.-H. Yaghmaee, "Improving on HEED protocol of wireless sensor networks using non probabilistic approach and fuzzy logic (HEEDNPF)," in Proceedings of the 5th International Symposium on Telecommunications (IST '10), vol. 2, pp. 193-198, Tehran, Iran, December 2010.

[13] F. Xiangning and S. H. Yulin, "Improvement on LEACH protocol of wireless sensor network," in Proceedings of the International Conference on Sensor Technologies and Applications (SENSORCOMM '07), pp. 260-264, Valencia, Spain, October 2007.

[14] E. Arbab, V. Aghazarian, and A. Hedayati, "A LEACH-based clustering algorithm for optimizing energy consumption," in Proceedings of the in 2nd International Conference on Computer Science and Information Technology (ICCSIT '12), vol. 2, pp. 147150, Singapore, April 2012.

[15] N. Sharma and V. Verma, "Energy efficient LEACH protocol for wireless sensor network," International Journal of Information and Network Security, vol. 2, no. 4, pp. 333-338, 2013.

[16] M. M. Umar, N. Alrajeh, and A. Mehmood, "SALMA: an efficient state-based hybrid routing protocol for mobile nodes in wireless sensor networks," International Journal of Distributed Sensor Networks, vol. 2016, Article ID 2909618, 11 pages, 2016.

[17] A. Kodole and P. Agarkar, "A survey of routing protocols in mobile ad hoc networks," Multidisciplinary Journal of Research in Engineering and Technology, vol. 2, no. 1, pp. 336-341, 2015.

[18] S. Roychowdhury and C. Patra, "Geographic adaptive fidelity and geographic energy aware routing in Ad-Hoc routing," in Proceedings of the International Conference (ACCTA '10), vol. 1, no. 2, pp. 309-313, August 2010. 
[19] B. Baranidharan and B. Shanthi, "A survey on energy efficient protocols for wireless sensor networks," International Journal of Computer Applications, vol. 11, pp. 35-40, 2010.

[20] F. Kiani, A. Rad, M. Sis, A. Kut, and A. Alpkocak, "EEAR: an energy effective-accuracy routing algorithm for wireless sensor networks," Life Science Journal, vol. 10, no. 2, pp. 39-45, 2013.

[21] S. D. Servetto and G. Barrenechea, "Constrained random walks on random graphs: routing algorithms for large scale wireless sensor networks," in Proceedings of the 1st ACM International Workshop on Wireless Sensor Networks and Applications, vol. 11, pp. 12-21, September 2002.

[22] G. Anastasi, M. Conti, M. Di Francesco, and A. Passarella, "Energy conservation in wireless sensor networks: a survey," Ad Hoc Networks, vol. 7, no. 3, pp. 537-568, 2009. 


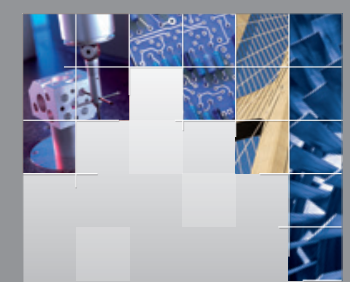

\section{Enfincering}
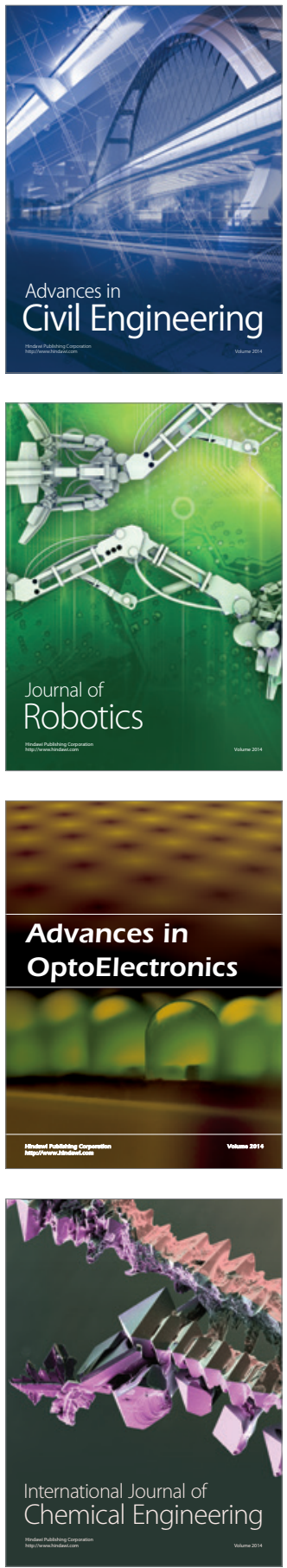

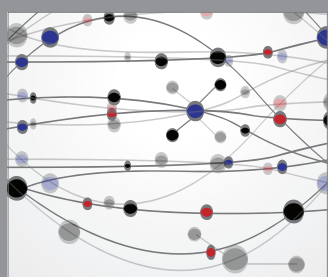

The Scientific World Journal

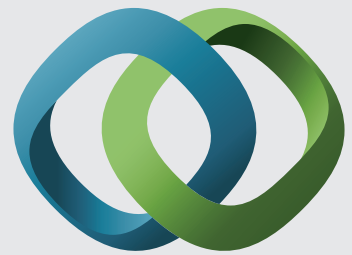

\section{Hindawi}

Submit your manuscripts at

http://www.hindawi.com
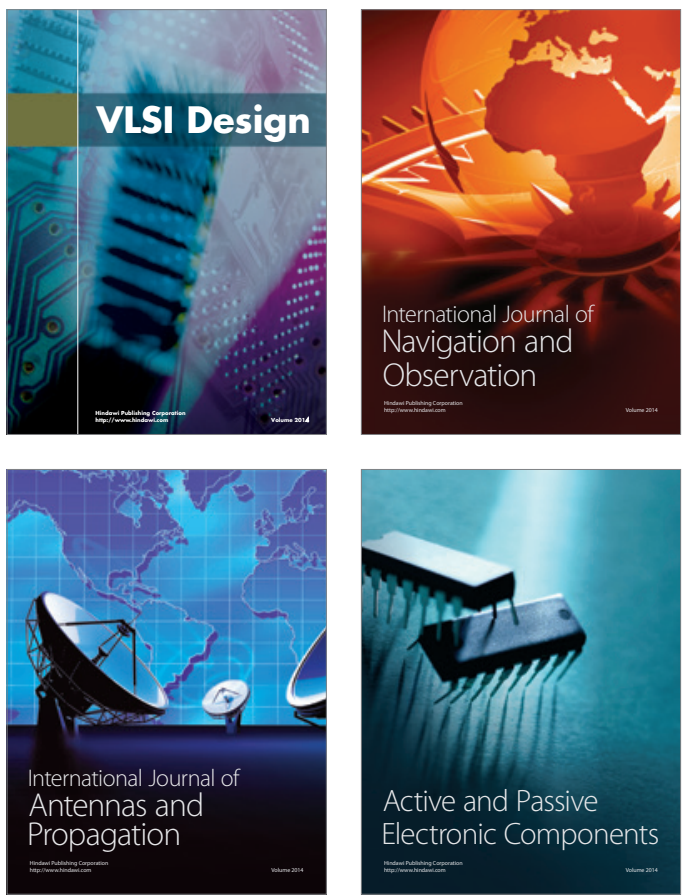
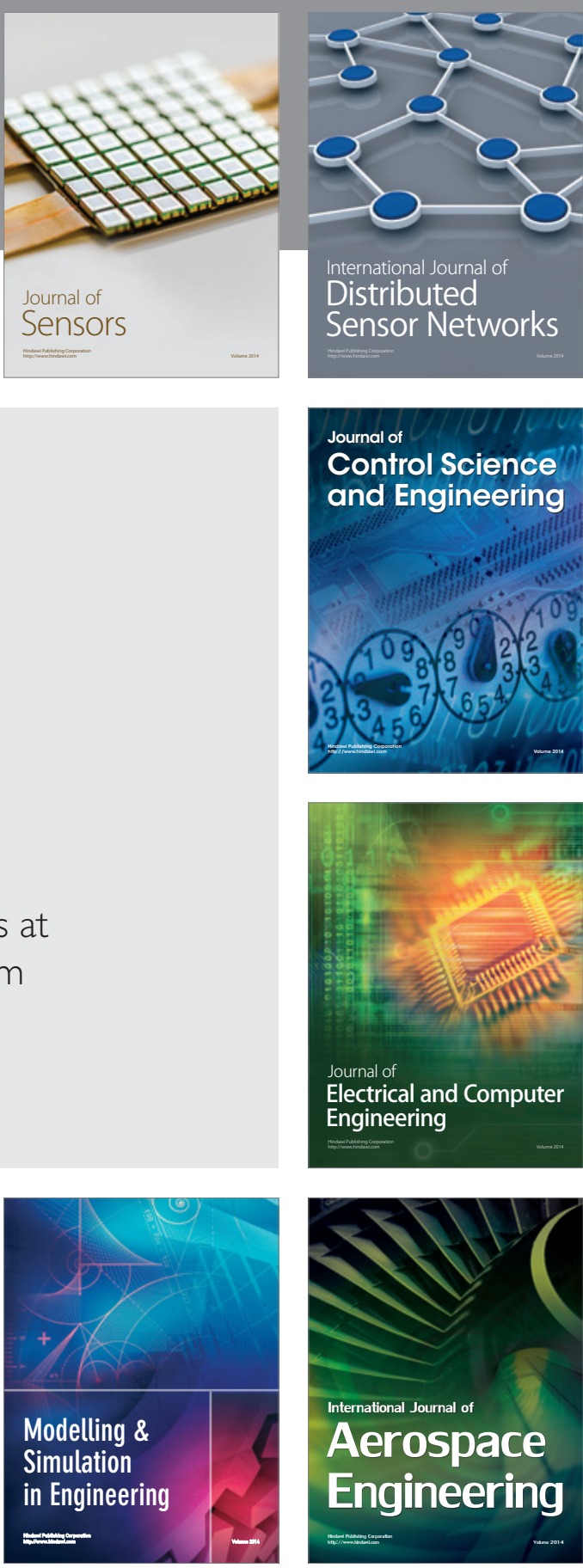

International Journal of

Distributed

Sensor Networks

Journal of

Control Science

and Engineering
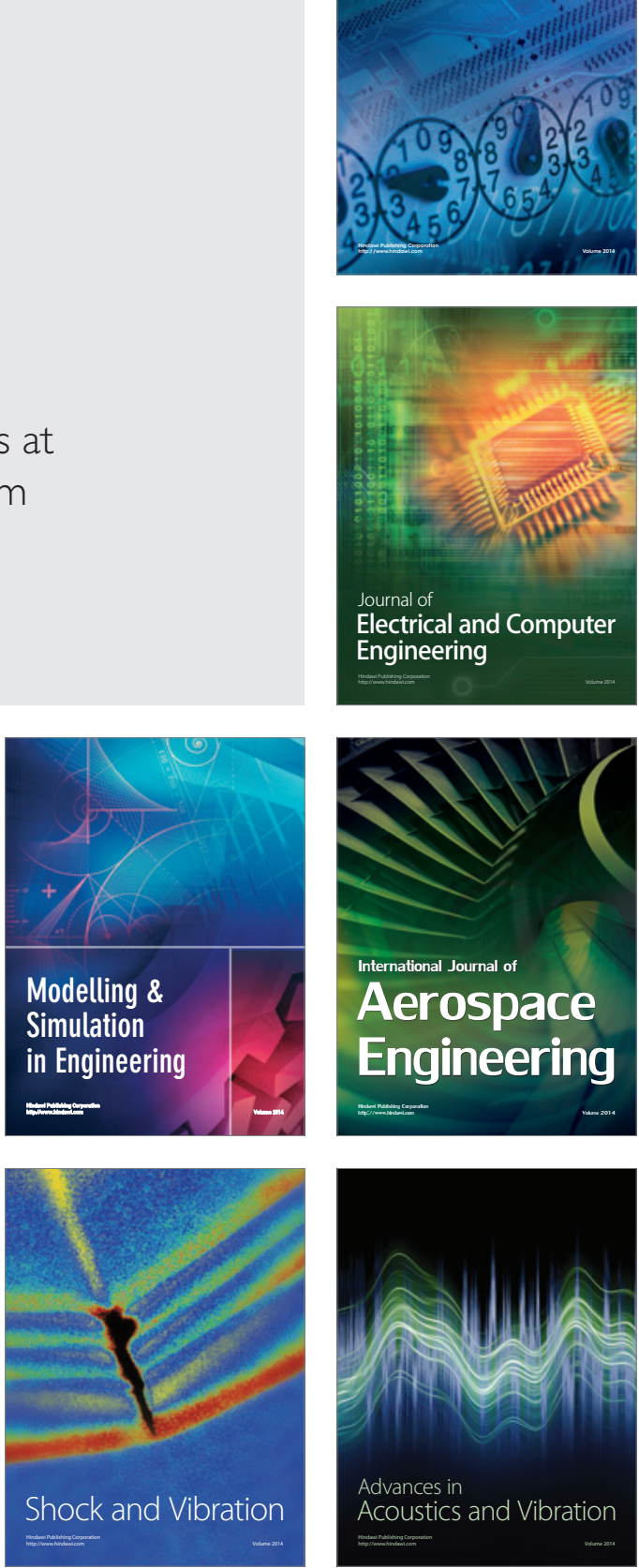